\title{
$5 \%$ Lidocaine-medicated plaster for the treatment of chronic peripheral neuropathic pain: complex regional pain syndrome and other neuropathic conditions
}

\author{
Enrique Calderón' \\ María Eloísa \\ Calderón-Seoane ${ }^{2}$ \\ Rafael García-Hernández' \\ Luis Miguel Torres' \\ 'Pain Unit, Anesthesiology \\ Department, Puerta del Mar \\ University Hospital, ${ }^{2}$ Health Center \\ North-Algeciras, Cadiz, Spain
}

\author{
This article was published in the following Dove Press journal: \\ Journal of Pain Research \\ 6 October 2016 \\ Number of times this article has been viewed
}

\begin{abstract}
Objectives: Chronic neuropathic pain and chronic complex regional pain syndrome (CRPS), in particular, are debilitating and difficult-to-treat conditions that have a strong impact on patient's quality of life. The aim of this study was to evaluate the effectiveness of $5 \%$ lidocaine-medicated plaster as add-on therapy in patients with chronic peripheral neuropathic pain conditions, including CRPS.
\end{abstract}

Patients and methods: This was a single-center, prospective, observational study set in a specialized pain unit of a tertiary hospital in Spain. A total of 56 patients with long-standing peripheral neuropathic pain, ten of them with CRPS, received 5\% lidocaine-medicated plaster as add-on analgesic therapy for 6 months.

Results: After 6 months of treatment, a $\geq 50 \%$ reduction in pain intensity was attained by $75 \%$ of patients, as measured by numeric rating scale (NRS) for pain. The average NRS score was reduced by $61 \%$ ( 4.7 points), from a baseline mean score of 7.8 to an end point mean score of 3.1. Marked improvements were also observed in the CRPS group: six out of ten patients achieved $\mathrm{a} \geq 50 \%$ reduction in NRS score, and the average NRS score for patients with CRPS was reduced by $51 \%$ ( 4.0 points), from a baseline mean score of 7.9 to an end point mean score of 3.9. The improvements in pain intensity were partially translated into a decrease in disability index and in anxiety levels.

Conclusion: 5\% Lidocaine-medicated plaster may be useful as add-on therapy for a number of peripheral neuropathic pain conditions, including CRPS.

Keywords: peripheral neuropathic pain, complex regional pain syndrome, topical lidocaine, analgesic therapy, chronic pain

\section{Introduction}

Neuropathic pain can be defined as "pain caused by a lesion or disease of the somatosensory system". ${ }^{1}$ It is a disabling condition that affects between $7 \%$ and $10 \%$ of the general population. ${ }^{2}$ While many cases of acute neuropathic pain have a benign course and resolve within a few months, in a proportion of patients the condition becomes chronic and persists for years, impairing physical functioning and severely affecting patient's quality of life. Neuropathic pain can be central (caused by a lesion or disease of the central somatosensory nervous system) or peripheral (caused by damage to peripheral somatosensory nervous system). It is estimated that in $\sim 60 \%$ of cases, the neuropathic pain is localized, ie, characterized by well-defined, circumscribed area(s) of maximum pain. ${ }^{3}$
Correspondence: Enrique Calderón Pain Unit, Anesthesiology Department, Puerta del Mar University Hospital, Av Ana de Viya, 21, 11009 Cadíz, Spain Tel +34956002897

Email ecalderons@gmail.com 
Complex regional pain syndrome (CRPS) is a rare and poorly understood neuropathic pain condition. It is usually associated with signs of autonomic and inflammatory changes: changes in skin color and temperature, sweating, edema, reduced strength, tremors, and dystonia. Perception can also be affected, reducing movement accuracy. Patients often experience serious impairments in activities of daily living and in ability to function. ${ }^{4}$

Pharmacological treatment of neuropathic pain, in general, and of CRPS, in particular, is challenging, with only few patients responding well to any given treatment. Conventional analgesics, such as nonsteroidal anti-inflammatory drugs (NSAIDs) and opioids, usually provide little relief, and the use of nonconventional analgesics (ie, antidepressants, anticonvulsants, and psychotropics) is common in clinical practice, ${ }^{5}$ although in case of CRPS the supporting clinical evidence is limited. ${ }^{5,6}$

Topical treatments can be an alternative or add-on therapy particularly useful in case of localized neuropathic pain. ${ }^{7}$ Their advantages, compared with systemic medications, include lower systemic dose, site-specific drug delivery, better tolerability, and avoidance of major drug-drug interactions, ${ }^{8}$ which can be particularly relevant in older patients.

In many countries, including Spain, 5\% lidocaine-medicated plaster is approved for treatment of postherpetic neuralgia (PHN). ${ }^{9}$ However, because of its effectiveness and safety in the management of PHN and its mechanism of action, lidocaine-medicated plaster is being increasingly used to treat other localized neuropathic pain conditions, such as postsurgical pain and painful diabetic polyneuropathy (DPN). ${ }^{8} \mathrm{Few}$ published case reports suggest that $5 \%$ lidocaine-medicated plaster might also be effective in CRPS, ${ }^{10,11}$ but the evidence so far has been anecdotal.

The objective of this study was to evaluate the effectiveness of 5\% lidocaine plaster as add-on therapy in patients with chronic peripheral neuropathic pain conditions, including CRPS.

\section{Patients and methods Study design}

This was a single-center, prospective, descriptive observational study set in the Pain Unit of the Department of Anesthesiology and Reanimation of a tertiary hospital in Cadiz, Spain. The study was carried out under the conditions of routine clinical practice, without modifications to prescribing patterns of the investigators. The study protocol was designed in agreement with the Declaration of Helsinki ${ }^{12}$ and the corresponding Spanish regulations and was approved by the ethical committee of Hospital Puerta del Mar.

\section{Patients}

Consecutive patients of both sexes who met the selection criteria were enrolled between March 2011 and September 2012. The inclusion criteria were age $\geq 18$ years, being diagnosed with a peripheral neuropathic pain syndrome, unsatisfactory control of pain with existing medication (numeric rating scale [NRS] score $\geq 6$ ), at least 6 months of pain duration, prescription of $5 \%$ lidocaine-medicated plaster on the day of the basal visit, and having signed the informed consent. The exclusion criteria were allergy or hypersensitivity toward amide-type anesthetics or ethers, pregnancy, or breastfeeding.

\section{Treatment}

At the basal visit, all the patients were prescribed 5\% lidocaine plaster as add-on therapy to apply once daily for up to 12 hours within a 24 -hour period. ${ }^{9}$

\section{Study procedures}

The study consisted of four visits (baseline, 1 month, 3 months, and 6 months). At baseline, the following data were collected: age, sex, medical history, actual analgesic treatment, Douleur Neuropathique 4 questions (DN4) questionnaire score for detection of neuropathic pain, ${ }^{13,14}$ pain intensity as measured by NRS for pain, ${ }^{15}$ baseline Oswestry disability index, ${ }^{16,17}$ and baseline Hospital Anxiety and Depression Scale (HAD; anxiety subscale of Hospital Anxiety and Depression Scale [HAD-A] and depression subscale of Hospital Anxiety and Depression Scale [HADD]) scores. ${ }^{18,19}$ At the following visits, the scales and the questionnaires (except DN4 questionnaire) were reapplied, and the patient and the investigator reported Patient's Global Impression of Improvement and Physician's Global Impression of Improvement. Concomitant treatments and adverse reactions were recorded at every visit.

NRS for pain is a patient-rated 11-point numeric scale with 0 representing "no pain" and 10 representing "worst pain imaginable".

The neuropathic pain DN4 questionnaire is a diagnostic tool that consists of ten items describing different pain characteristics. A score of at least four of ten possible points is considered to identify neuropathic pain with $83 \%$ sensitivity and $90 \%$ specificity.

The HAD-A and HAD-D are the two parts of the HAD scale. HAD-A explores anxiety levels, whereas HAD-D explores depression levels. Each of the scales represents a questionnaire consisting of seven questions, and the score ranges from 0 (no anxiety or depression) to 21 (maximal levels of anxiety or depression). Generally, a score of 8 points or more is considered as a cutoff for diagnosis of anxiety or depression. ${ }^{20}$ 
The Oswestry disability index is an index derived from the Oswestry Low Back Pain Questionnaire, which was developed to quantify disability caused by low back pain. The index ranges from $0 \%$ (no disability) to $100 \%$ (total disability).

Patient's Global Impression of Improvement and Physician's Global Impression of Improvement are subjective estimations of the overall change in the patient's health state relative to the basal visit given by the patient and the investigator, respectively.

\section{Statistical methods}

Since the study had an exploratory character, no formal sample size calculation was carried out. The number of participants was estimated based on feasibility and condition prevalence. Descriptive statistics were used to summarize basal characteristics. Differences in characteristics between groups were assessed using the Kruskal-Wallis or Fisher's exact test. Intrapatient score changes were analyzed using the Wilcoxon signed-rank test. Differences in the extent of score changes between the patient groups were analyzed using the Mann-Whitney $U$ test. Strength of correlation between variables was characterized by Kendall rank correlation coefficient $\left(\tau_{\mathrm{b}}\right)$.

\section{Results}

\section{Patient disposition and basal characteristics}

Sixty patients were recruited in the study. Data for four patients were excluded from the analysis because the patients did not have peripheral neuropathic pain but central neuropathic pain. All the participants completed the study.
The patients were between 28 years and 91 years old, and the majority were females $(34 / 56)$. To assess the effectiveness of analgesic treatment in different peripheral neuropathic pain conditions, patients were categorized into five groups according to the diagnosis: CRPS $(10 / 56,18 \%)$, PHN (8/56, 14\%), DPN (7/56, 12\%), chronic postsurgery pain (CPSP, 16/56, 29\%), and nerve entrapment syndrome (NES, 15/56, 27\%).

There were few differences in the basal characteristics between the groups (Table 1). Statistically significant differences were found in age, with patients with PHN or with DPN being on average older than the rest of the patients. Variations in male/female ratio among the groups were not statistically significant.

At baseline, the patients were receiving between one and five concomitant treatments (median 2), which included anticonvulsants (75\%), antidepressants (59\%), NSAIDs (27\%), opioids (48\%), and advanced analgesic treatments, such as nerve blocks and radiofrequency (16\%). The number of concomitant treatments was similar between the groups (Table 1). The data on concomitant treatments were summarized using WHO's modified analgesic ladder: ${ }^{21}$ Only adjuvants (antidepressants and/or anticonvulsants); step I - nonopioid analgesic drugs (NSAIDs) \pm adjuvants; step II - opioids for mild to moderate pain \pm nonopioids \pm adjuvants; step III - opioids for moderate to severe pain \pm nonopioids \pm adjuvants; and step IV - advanced analgesic therapies (nerve blocks, radiofrequency, and intravenous lidocaine) \pm the abovementioned treatments. There was no statistically significant association between the diagnosis and the analgesic ladder step.

Table I Baseline demographic and clinical characteristics of patients

\begin{tabular}{|c|c|c|c|c|c|c|}
\hline Variable & All patients, $n=56$ & CRPS, $n=10$ & PHN, $n=8$ & DPN, $n=7$ & CPSP, $n=16$ & NES, $n=15$ \\
\hline Age (years), mean (SD) & $58.3(14.9)$ & $53.0(15.2)$ & $67.5(11.6)$ & $68.3(6.5)$ & $53.1(15.8)$ & $57.8(I 5.0)$ \\
\hline Females, n (\%) & $34(60.7)$ & $5(50.0)$ & $5(62.5)$ & $3(42.8)$ & II (68.8) & $10(66.7)$ \\
\hline Months since diagnosis, mean (SD) & $8.9(2.7)$ & $8.9(2.6)$ & $8.0(1.7)$ & $12.1(2.8)$ & $8.7(3.2)$ & $8.1(1.6)$ \\
\hline Number of concomitant treatments, mean (SD) & $2.3(0.8)$ & $2.1(0.8)$ & $2.3(0.5)$ & $2.4(0.5)$ & $2.5(1.2)$ & $2.1(0.6)$ \\
\hline \multicolumn{7}{|l|}{ Analgesic ladder step, $\mathrm{n}(\%)$} \\
\hline Only adjuvants & $23(4 I)$ & $4(40.0)$ & $5(62.5)$ & $3(42.9)$ & $4(25.0)$ & $7(46.6)$ \\
\hline Step I & $4(7.1)$ & $I(10.0)$ & $0(0)$ & $0(0)$ & I (6.3) & $2(13.3)$ \\
\hline Step II & $13(23.2)$ & $3(30.0)$ & I (I2.5) & $2(28.6)$ & $3(18.8)$ & $4(26.7)$ \\
\hline Step III & $6(10.7)$ & $I(10.0)$ & $\mathrm{I}(12.5)$ & I (I4.3) & $2(12.5)$ & I (6.7) \\
\hline Step IV & $10(17.8)$ & $I(10.0)$ & $I(12.5)$ & $\mathrm{I}(\mathrm{I} 4.3)$ & $6(37.5)$ & I (6.7) \\
\hline DN4 score, mean (SD) & $7.4(1.2)$ & $8.2(0.9)$ & $7.8(1.2)$ & $6.4(0.8)$ & $7.5(1.2)$ & $7.1(1.3)$ \\
\hline NRS score, mean (SD) & $7.8(1.1)$ & $7.9(0.7)$ & $8.8(1.0)$ & $7.4(1.3)$ & $7.4(1.0)$ & $7.7(1.0)$ \\
\hline Oswestry disability index, mean (SD) & $24.5(12.4)$ & $27.7(15.7)$ & $22.5(13.9)$ & $17.9(8.7)$ & $25.8(13.7)$ & $25.3(9.1)$ \\
\hline HAD-A, mean (SD) & $6.3(3.0)$ & $66.3(3.6)$ & $6.3(2.6)$ & $4.4(1.3)$ & $7.7(3.2)$ & $5.7(2.9)$ \\
\hline HAD-D, mean (SD) & $5.8(3.8)$ & $4.8(3.3)$ & $6.4(2.6)$ & $6.6(2.6)$ & $6.3(4.2)$ & $5.4(4.7)$ \\
\hline
\end{tabular}

Abbreviations: CPSP, chronic postsurgery pain; CRPS, complex regional pain syndrome; DN4, Douleur Neuropathique 4 questions; DPN, diabetic polyneuropathy; HAD-A, anxiety subscale of Hospital Anxiety and Depression Scale; HAD-D, depression subscale of Hospital Anxiety and Depression Scale; NES, nerve entrapment syndrome; NRS, numeric rating scale; PHN, postherpetic neuropathy. 
The DN4 score for neuropathic pain detection was high for all the patients (median 7, range 6-10) and showed modest but statistically significant variations between the groups $(P=0.015)$. The DN4 score was higher in the CRPS group (range 6-10, median 8.0) than in the rest of the patients ( $P=0.016$ when compared with the rest of the patients).

The basal scores for NRS, Oswestry disability index, HAD-D, and HAD-A were similar between the groups (Table 1). Most patients reported minimal or moderate disability as measured by the Oswestry index $(\leq 40 \%$ disability), and seven (12.5\%) patients (two in the CRPS groups, three in the CPSP group, one in the NES group, and one in the PHN group) reported severe disability. Twenty (35.7\%) patients had a HAD-A score of $\geq 8$, corresponding to clinical anxiety, and $21(37.5 \%)$ patients had a HAD-D score of $\geq 8$, corresponding to clinical depression.

\section{Main results}

Over 6 months of treatment, all patients experienced a decrease in pain intensity, as measured by NRS (Figure 1 and Table 2). All patients, except for two patients in the CRPS group, reported at least some improvement in pain already after 1 month of treatment. NRS score continued to decrease in the subsequent visits (Figure $1 ; P<0.001$ in the Wilcoxon signed-rank test when comparing NRS scores of any visit with the baseline score or with the previous visit score). After

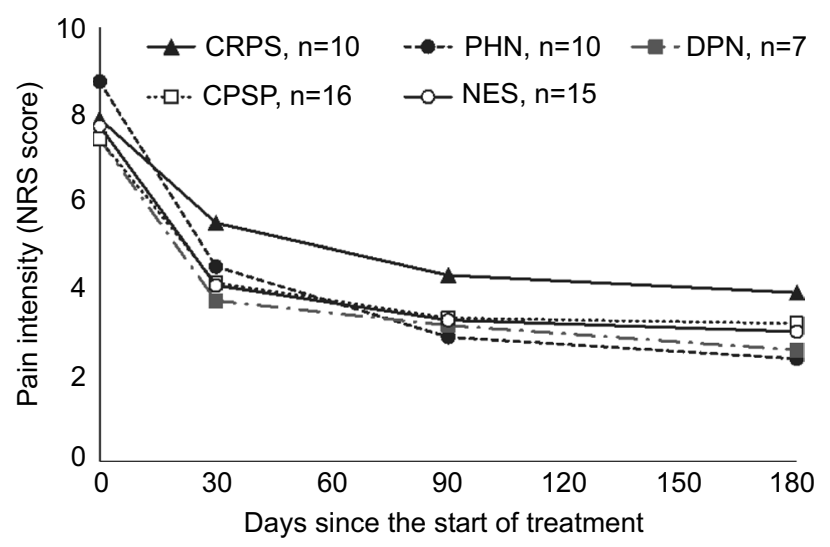

Figure I Change in average NRS over time by clinical entity.

Abbreviations: CPSP, chronic postsurgery pain; CRPS, complex regional pain syndrome; DPN, diabetic polyneuropathy; NES, nerve entrapment syndrome; NRS, numeric rating scale; $\mathrm{PHN}$, postherpetic neuropathy

Table 2 Treatment outcomes for the overall sample and by clinical entity

\begin{tabular}{|c|c|c|c|c|c|c|}
\hline Variable & All patients, $n=56$ & CRPS, $n=10$ & PHN, $n=8$ & DPN, $n=7$ & CPSP, $n=16$ & $\begin{array}{l}\text { NES, } \\
n=15\end{array}$ \\
\hline \multicolumn{7}{|c|}{ Change in intrapatient NRS score from baseline, mean (SD) } \\
\hline At I month & $-3.4(1.7)$ & $-2.4(1.7)$ & $-4.3(1.6)$ & $-3.7(0.8)$ & $-3.3(1.5)$ & $-3.7(2.1)$ \\
\hline At 3 months & $-4.4(1.7)$ & $-3.6(2.0)$ & $-5.9(1.5)$ & $-4.3(0.8)$ & $-4.1(1.2)$ & $-4.5(2.1)$ \\
\hline At 6 months & $-4.7(1.9)$ & $-4.0(2.3)$ & $-6.3(1.8)$ & $-4.9(1.2)$ & $-4.3(1.4)$ & $-4.7(2.0)$ \\
\hline \multicolumn{7}{|c|}{ Oswestry index change from baseline at month 6 among patients, $n(\%)^{a}$} \\
\hline$>0 \%$ & $7(12.5)$ & I (I0.0) & $0(0)$ & $3(42.9)$ & I (6.3) & $2(13.3)$ \\
\hline $0 \%$ & $14(25.0)$ & $2(20.0)$ & $3(37.5)$ & I (I4.3) & $5(3 \mid .3)$ & $3(20.0)$ \\
\hline$-1 \%$ to $-10 \%$ & $32(57.1)$ & $6(60.0)$ & $4(50.0)$ & $3(42.9)$ & $9(56.3)$ & $10(66.7)$ \\
\hline$<-10 \%$ & $3(5.4)$ & I (I0.0) & I (I2.5) & $0(0)$ & I (6.3) & $0(0)$ \\
\hline \multicolumn{7}{|c|}{ HAD-A score reduction from baseline at month 6 among patients, $n$ (\%) } \\
\hline 0 points & $26(46.4)$ & $6(60.0)$ & $2(25.0)$ & $5(7 I .4)$ & $7(43.8)$ & $6(40.0)$ \\
\hline $1-2$ points & $17(30.4)$ & I (I0.0) & $5(62.5)$ & $0(0)$ & $5(3 \mid .3)$ & $6(40.0)$ \\
\hline$\geq 3$ points & $13(23.1)$ & $3(30.0)$ & I (I2.5) & $2(28.6)$ & $4(25.1)$ & $3(20.0)$ \\
\hline \multicolumn{7}{|c|}{ HAD-D score reduction from baseline at month $6, \mathrm{n}(\%)^{\mathrm{b}}$} \\
\hline 0 points & $42(75.0)$ & $6(60.0)$ & $5(62.5)$ & $7(100.0)$ & $13(81.3)$ & $10(66.7)$ \\
\hline I-2 points & $8(14.0)$ & $2(20.0)$ & $2(25.0)$ & $0(0)$ & $2(12.5)$ & $3(20.0)$ \\
\hline$\geq 3$ points & $5(8.7)$ & I (I0.0) & $\mathrm{I}(12.5)$ & $0(0)$ & I (6.3) & $2(13.4)$ \\
\hline Patient's Global Impression of & $37.9 \%(19.4 \%)$ & $34.0 \%(23.7 \%)$ & $43.1 \%(24.3 \%)$ & $35.0 \%(14.7 \%)$ & $38.4 \%(20.7 \%)$ & $38.7 \%$ \\
\hline Improvement after 6 months, mean (SD) & & & & & & $(15.5 \%)$ \\
\hline Physician's Global Impression of & $38.6 \%(18.9 \%)$ & $35.0 \%(23.1 \%)$ & $43.1 \%(21.9 \%)$ & $35.0 \%(14.7 \%)$ & $40.0 \%(20.8 \%)$ & $38.7 \%$ \\
\hline Improvement after 6 months, mean (SD) & & & & & & $(15.5 \%)$ \\
\hline \multicolumn{7}{|c|}{ Patients with changes in concomitant analgesic treatments at 6 months, $n$ (\%) } \\
\hline No changes from baseline & $20(35.7)$ & $3(30.0)$ & $4(50.0)$ & $3(42.6)$ & $3(18.8)$ & $7(46.7)$ \\
\hline Dose of I or 2 analgesics reduced & $29(51.7)$ & $6(60.0)$ & $3(37.5)$ & $4(57.1)$ & II (68.8) & $6(40.0)$ \\
\hline Number of analgesic treatments reduced & $6(10.7)$ & I (10.0) & I (I2.5) & $0(0)$ & $2(12.5)$ & $2(13.3)$ \\
\hline
\end{tabular}

Notes: a Negative change in Oswestry index indicates reduction in disability. ${ }^{\mathrm{b}}$ The HAD-D score increased from baseline at month 6 in one patient in the CRPS group. Abbreviations: CPSP, chronic postsurgery pain; CRPS, complex regional pain syndrome; DPN, diabetic polyneuropathy; HAD-A, anxiety subscale of Hospital Anxiety and Depression Scale; HAD-D, depression subscale of Hospital Anxiety and Depression Scale; NES, nerve entrapment syndrome; NRS, numeric rating scale; PHN, postherpetic neuropathy. 
6 months of treatment, the average NRS score was reduced by $60.6 \%$ ( 4.7 points), from a baseline mean score of 7.8 to an end point mean score of 3.1. Importantly, there were no cases of loss of effectiveness during the study.

The overall percentage of patients who experienced at least $50 \%$ improvement in pain intensity (50\% responders) was $46.4 \%$ after 1 month of treatment, $71.4 \%$ after 3 months of treatment, and reached $75 \%$ by 6 months of treatment (Figure 2). The percentage of responders was numerically higher in patients with DPN (100\% at 6 months) or PHN (88\% at 6 months) and somewhat lower in patients with CRPS, NES, and CPSP $(60 \%, 66.7 \%$, and $75 \%$ at 6 months, respectively), although the differences were not statistically significant.

The decrease in pain intensity was partially translated into improvements in functioning (Table 2). Thus, 35 (62.5\%) patients after 6 months of treatment reported a reduction in the Oswestry disability index, and in three (5.4\%) patients this reduction was $\geq 10$ percentage points. In seven $(12.5 \%)$ patients, the Oswestry disability index increased after 6 months (by $<5$ percentage points in all cases), and in $14(25.0 \%)$ patients it did not change. The number of patients with severe disability (Oswestry disability index $>40 \%$ ) reduced from seven $(12.5 \%)$ patients to two $(3.6 \%)$ patients. However, there was only a weak correlation between baseline Oswestry disability index and baseline NRS score $\left(\tau_{\mathrm{b}}=0.26, P=0.014\right)$, and there was no significant correlation between changes in NRS score and changes in Oswestry index, suggesting that a large component of disability was other than pain.
As for the changes in psychiatric aspects, 30 (53.6\%) patients reported a decrease in their anxiety levels, as measured by HAD-A, whereas $26(46.3 \%)$ reported no change after 6 months of treatment (Table 2). The number of patients diagnosed with anxiety (HAD-A $\geq 8$ ) decreased from 20 (35.7\%) at baseline to $12(21.4 \%)$ after 6 months of treatment.

Depression levels decreased after 6 months in 13 (23\%) patients, as assessed by HAD-D, whereas in $42(75 \%)$ patients the levels did not change, and in one $(1.8 \%)$ patient the HAD-D score increased. The number of patients diagnosed with depression (HAD-D $\geq 8$ ) decreased from 21 (37.5\%) at baseline to $14(25.0 \%)$ after 6 months of treatment.

All patients except one reported overall improvement in their condition (Patient's Global Impression of Improvement), with $48(85.7 \%)$ patients assessing their improvement after 6 months of treatment as $\geq 25 \%$ compared with the baseline and with $15(26.8 \%)$ patients reporting an overall improvement of $\geq 50 \%$. Similarly, the Physician's Global Impression of Improvement was positive for all the patients, was $\geq 25 \%$ in $48(85.7 \%)$ patients, and $\geq 50 \%$ in $13(23.2 \%)$ patients. There was a strong correlation between the global impressions of improvement given by the patient and by the physician $\left(\tau_{\mathrm{b}}=0.93, P<0.001\right)$. Both Patient's Global Impression of Improvement and Physician's Global Impression of Improvement showed a statistically significant negative correlation with changes in NRS score $\left(\tau_{\mathrm{b}}=-0.37, P<0.001\right.$ and $\tau_{\mathrm{b}}=-0.35, P<0.001$, respectively).

Changes observed in patients with CRPS were similar to those observed in the rest of the patients. The average change

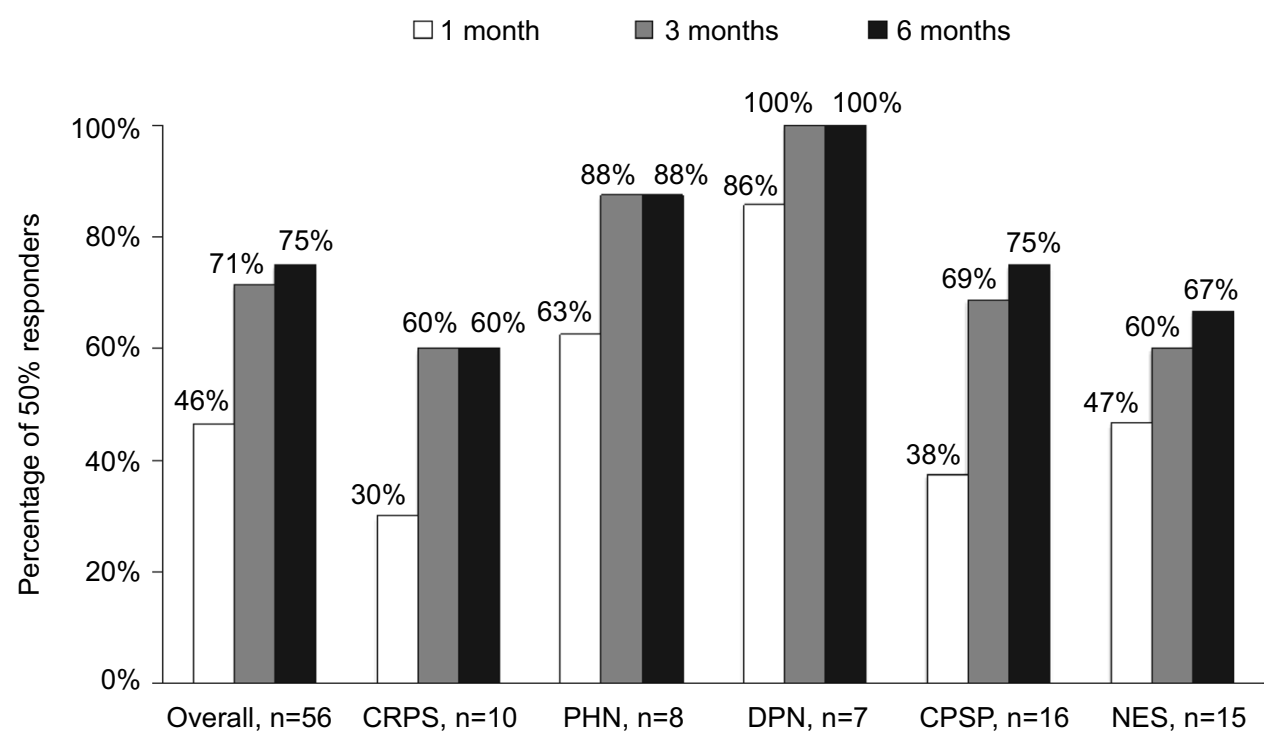

Figure 2 The percentage of patients reporting at least 50\% pain intensity reduction, as measured by NRS score, after I month, 3 months, and 6 months of treatment. Abbreviations: CPSP, chronic postsurgery pain; CRPS, complex regional pain syndrome; DPN, diabetic polyneuropathy; NES, nerve entrapment syndrome; NRS, numeric rating scale; $\mathrm{PHN}$, postherpetic neuropathy. 
in NRS score compared with baseline was numerically smaller in patients with CRPS than in patients in the other groups, although the difference was not statistically significant (Figure 1 and Table 2). Nevertheless, after 6 months of treatment the average NRS score in the CRPS group was reduced by $50.6 \%$ (4.0 points) from a baseline mean score of 7.9 to an end point mean score of 3.9. Six (60\%) patients in the CRPS groups experienced a reduction of at least $50 \%$ in pain intensity after 6 months of treatment.

Nine (90\%) patients with CRPS had an impression that their overall condition improved (Patient's Global Impression of Improvement), with two (20\%) of them estimating their improvement as $<25 \%$, four (40\%) estimating their improvement as at least $25 \%$ but $<50 \%$, and three $(30 \%)$ patients reported an improvement of $\geq 50 \%$. The Physician's Global Impression of Improvement was concordant with the patient's impressions.

Correlation analysis revealed no association between changes in scales scoring, on one hand, and baseline patient characteristics (sex, age, or baseline analgesic step), with one exception. Namely, there was a weak but statistically significant negative correlation $\left(\tau_{\mathrm{b}}=-0.21, P=0.03\right)$ between changes in NRS score and patient age (greater changes were observed in older patients). However, this observation is difficult to interpret, since age distribution was not uniform between the diagnosis groups, and the groups that included older patients (DPN and PHN) happened to be those that had the most pronounced changes in NRS score.

After 6 months, the number of concomitant analgesic treatments could be reduced in six $(10.7 \%)$ patients, while in $29(51.7 \%)$ patients the dose of at least one of the concomitant analgesic treatments could be reduced (Table 2). There were no cases of new analgesic treatments added during the course of the study.

Four $(7.1 \%)$ patients reported adverse effects that the investigator considered related to the use of 5\% lidocainemedicated plaster. All of these side effects were limited to local skin reactions (itchiness, redness, or dry skin) and did not require treatment discontinuation.

\section{Discussion}

In many countries, including Spain, 5\% lidocaine-medicated plaster is currently approved only for treating a particular type of neuropathic pain, the postherpetic neuralgia. ${ }^{9}$ However, an increasing body of clinical evidence suggests that $5 \%$ lidocaine-medicated plaster may be effective for treating other types of peripheral neuropathic pain. ${ }^{8}$ Some authors proposed algorithms for identifying and treating patients with localized neuropathic pain, in which topical agents, such as $5 \%$ lidocaine plasters, are incorporated as cornerstones. ${ }^{7,22}$ A recent Cochrane review concluded that there was "no evidence from good quality randomized controlled studies to support the use of topical lidocaine to treat neuropathic pain, although individual studies indicated that it was effective for relief of pain". ${ }^{23}$ However, most studies on effectiveness of topical lidocaine in peripheral neuropathic pain were focused on PHN, DPN, postsurgical, and posttraumatic pain. Until now, to our knowledge, only few case reports suggested potential usefulness of lidocaine plasters in patients with CRPS.

The present study, which was set in a specialized pain unit in a tertiary hospital, provides further insight into the usefulness of 5\% lidocaine-medicated plasters in patients with chronic peripheral neuropathic pain. A rapid marked improvement in pain intensity was observed in most patients across all the groups, including patients with CRPS. The response was numerically higher in the PHN and DPN groups and numerically lower in the CRPS group, both for the average change in NRS score and for the proportion of $50 \%$ responders. However, it is hard to appreciate the statistical significance of these differences due to the low number of patients.

Interestingly, in all groups the average pain intensity continued to decrease after 1 month, and the number of $50 \%$ responders plateaued after 3 months of treatment (Figures 1 and 2). This suggests that some patients respond to treatment in a gradual and progressive manner, and that the treatment should not be discontinued too prematurely in case of slow response.

The observed decrease in pain intensity only partially translated into improvements in functioning. However, given the diversity of the included diagnoses, the choice of the assessment tools was not trivial, and the selected tool (Oswestry disability index) might have not been sensitive enough to detect changes associated with the particular painful conditions. Similarly, a proportion of patients showed an improvement in their psychological well-being, as measured by HAD-A and HAD-D.

Lidocaine-medicated plasters were well tolerated, as demonstrated by low rate of side effects and absence of plaster discontinuation.

Importantly, 5\% lidocaine-medicated plaster has been shown to be useful as an add-on therapy in patients with long-standing CRPS. Although this group was composed of only ten patients, to our knowledge this is the largest reported series of patients with long-standing CRPS treated with 
topical lidocaine. This syndrome is one of the most debilitating and hard-to-treat neuropathic pain conditions. It is rare, and there are only a few published reports on the effects of analgesic treatments in CRPS. A recent systematic review on the interventions in CRPS found low-quality evidence suggesting efficacy of only few pharmacological treatments (bisphosphonates, calcitonin, and intravenous ketamine) in pain reduction in CRPS. ${ }^{6}$

This study has several limitations. First, this was an observational, noncontrolled study. The topical treatment was added to the previous analgesic treatments, and it is impossible to appreciate the contribution of each treatment to the observed pain relief, as well as the contribution of the natural course of the conditions. However, it is important to bear in mind that the study population was composed of patients with long-standing neuropathic pain conditions (>6 months) who did not obtain satisfactory results with previous analgesic therapy, and that it is difficult to achieve significant improvements in pain intensity in this population.

Other limitations of the study include the small sample size, absence of more specific tools to evaluate disability and quality of life associated with chronic neuropathic pain, lack of recorded information on specific symptoms of neuropathic pain, and the intrinsic weaknesses of NRS. Despite being a widely accepted, reliable, sensitive, and specific tool for assessing pain, NRS only evaluates the intensity of pain and does not capture the complexity of neuropathic pain experience. ${ }^{24}$

On the other hand, the strengths of this study include its real-life character, which allows cautious generalization of the results. As mentioned earlier, patients with chronic neuropathic pain referred to specialized pain units are difficult to treat. They tend to be polymedicated, similar to the population of the present study, with a high rate of analgesia-related side effects. ${ }^{25}$ The present data suggest that 5\% lidocainemedicated plaster may be useful as add-on therapy in patients with persistent peripheral neuropathic pain and may allow for reduction of systemic analgesic treatments and related side effects.

An additional strength of this study is its long duration (6 months), which is highly relevant in the context of the chronicity of the studied conditions. This allowed two important observations: first, that there were no cases of loss of effectiveness $>6$ months, and second, that the lidocaine plasters were well tolerated even over such a long treatment period.

\section{Conclusion}

Altogether, these data suggest that 5\% lidocaine plaster may be useful as add-on therapy in patients with chronic peripheral neuropathic pain conditions. The observed improvement in patients with CRPS may open a new therapeutic strategy in management of this complex and challenging condition. Further high-quality controlled studies to corroborate the efficacy and effectiveness of topical lidocaine in CRPS are warranted.

\section{Acknowledgments}

The authors thank Ansgar Seyfferth and STAT-UP Statistical Consulting \& Data Science Services for the help with statistical analysis, and Zhanna Shcheprova and Content Ed Net for assistance in writing and editing the manuscript. This was an independent study. These data have not been presented previously.

\section{Disclosure}

Dr Calderon has received compensations for consulting services, speaking activities and travel/accommodations/ meeting expenses support from Gebro, Astellas, Abbott, Grunenthal and Ferrer. Dr Garcia-Hernandez has received compensations for speaking activities and travel/accommodations/meeting expenses support from Orion, Baxter, MSD, Braun and Astellas. Dr Calderon-Seoane has received compensations for meeting expenses support from Grünenthal. Dr Torres has received compensations for consulting services, scientific advisory boards, speaking activities and travel/accommodations/ meeting expenses support from Pfizer, Abbott, Astellas, Mundipharma, Grünenthal, Takeda, Menarini and Cardiva.

\section{References}

1. Jensen TS, Baron R, Haanpaa M, et al. A new definition of neuropathic pain. Pain. 2011;152(10):2204-2205.

2. van Hecke O, Austin SK, Khan RA, Smith BH, Torrance N. Neuropathic pain in the general population: a systematic review of epidemiological studies. Pain. 2014;155(4):654-662.

3. Mick G, Baron R, Finnerup NB, et al. What is localized neuropathic pain? A first proposal to characterize and define a widely used term. Pain Manag. 2012;2(1):71-77.

4. Bruehl S. Complex regional pain syndrome. BMJ. 2015;351:h2730.

5. Finnerup NB, Attal N, Haroutounian S, et al. Pharmacotherapy for neuropathic pain in adults: a systematic review and meta-analysis. Lancet Neurol. 2015;14(2):162-173.

6. O'Connell NE, Wand BM, McAuley J, Marston L, Moseley GL. Interventions for treating pain and disability in adults with complex regional pain syndrome. Cochrane Database Syst Rev. 2013;(4):CD009416.

7. Casale R, Mattia C. Building a diagnostic algorithm on localized neuropathic pain (LNP) and targeted topical treatment: focus on 5\% lidocaine-medicated plaster. Ther Clin Risk Manag. 2014;10:259-268.

8. Mick G, Correa-Illanes G. Topical pain management with the $5 \%$ lidocaine medicated plaster - a review. Curr Med Res Opin. 2012;28(6):937-951.

9. Versatis(R). [Ficha técnica]. Aachen, Germany: Gruenethal Pharma; 2016.

10. Frost SG. Treatment of complex regional pain syndrome type 1 in a pediatric patient using the lidocaine patch 5\%: a case report. Curr Ther Res Clin Exp. 2003;64(8):626-629.

11. Karmarkar A, Lieberman I. Management of complex regional pain syndrome type II using lidoderm 5\% patches. Br J Anaesth. 2007; 98(2):261-262. 
12. World Medical A. World Medical Association declaration of Helsinki: ethical principles for medical research involving human subjects. JAMA. 2013;310(20):2191-2194.

13. Bouhassira D, Attal N, Fermanian J, et al. Development and validation of the neuropathic pain symptom inventory. Pain. 2004;108(3): 248-257.

14. Perez C, Galvez R, Huelbes S, et al. Validity and reliability of the Spanish version of the DN4 (Douleur Neuropathique 4 questions) questionnaire for differential diagnosis of pain syndromes associated to a neuropathic or somatic component. Health Qual Life Outcomes. 2007;5:66.

15. Downie WW, Leatham PA, Rhind VM, Wright V, Branco JA, Anderson JA. Studies with pain rating scales. Ann Rheum Dis. 1978;37(4):378-381.

16. Fairbank JC, Couper J, Davies JB, O'Brien JP. The Oswestry low back pain disability questionnaire. Physiotherapy. 1980;66(8):271-273.

17. Payares K, Lugo LH, Morales V, Londono A. Validation in Colombia of the Oswestry disability questionnaire in patients with low back pain. Spine (Phila Pa 1976). 2011;36(26):E1730-E1735.

18. Zigmond AS, Snaith RP. The hospital anxiety and depression scale. Acta Psychiatr Scand. 1983;67(6):361-370.

19. Herrero MJ, Blanch J, Peri JM, De Pablo J, Pintor L, Bulbena A. A validation study of the hospital anxiety and depression scale (HADS) in a Spanish population. Gen Hosp Psychiatry. 2003;25(4):277-283.
20. Bjelland I, Dahl AA, Haug TT, Neckelmann D. The validity of the Hospital Anxiety and Depression Scale. An updated literature review. J Psychosom Res. 2002;52(2):69-77.

21. Vargas-Schaffer G. Is the WHO analgesic ladder still valid? Twenty-four years of experience. Can Fam Physician. 2010;56(6): e202-e515.

22. Allegri M, Baron R, Hans G, et al. A pharmacological treatment algorithm for localized neuropathic pain. Curr Med Res Opin. 2016;32(2):377-384.

23. Derry S, Wiffen PJ, Moore RA, Quinlan J. Topical lidocaine for neuropathic pain in adults. Cochrane Database Syst Rev. 2014;(7): CD010958.

24. Hawker GA, Mian S, Kendzerska T, French M. Measures of adult pain: Visual Analog Scale for Pain (VAS Pain), Numeric Rating Scale for Pain (NRS Pain), McGill Pain Questionnaire (MPQ), Short-Form McGill Pain Questionnaire (SF-MPQ), Chronic Pain Grade Scale (CPGS), Short Form-36 Bodily Pain Scale (SF-36 BPS), and Measure of Intermittent and Constant Osteoarthritis Pain (ICOAP). Arthritis Care Res (Hoboken). 2011;63(supp1 11):S240-S252.

25. Moulin DE, Clark AJ, Gordon A, et al. Long-term outcome of the management of chronic neuropathic pain: a prospective observational study. J Pain. 2015;16(9):852-861.
Journal of Pain Research

\section{Publish your work in this journal}

The Journal of Pain Research is an international, peer reviewed, open access, online journal that welcomes laboratory and clinical findings in the fields of pain research and the prevention and management of pain. Original research, reviews, symposium reports, hypothesis formation and commentaries are all considered for publication.

\section{Dovepress}

The manuscript management system is completely online and includes a very quick and fair peer-review system, which is all easy to use. Visit http://www.dovepress.com/testimonials.php to read real quotes from published authors. 